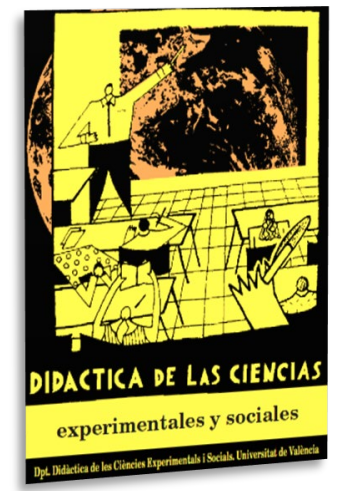

\title{
La alfabetización científica en estudiantes de Formación Profesional Básica a través de una secuencia adaptada hacia la inclusión educativa
}

\author{
Scientific literacy in Basic Vocational Education \\ and Training students through an adapted \\ sequence towards educational inclusion
}

DOI: $10.7203 / D C E S .41 .20872$

\author{
Sandra López Santos \\ Universidad de Huelva, sandra.santos@alu.uhu.es \\ ORCID iD: https://orcid.org/0000-0002-7825-003X \\ María de los Ángeles De las Heras Pérez \\ Universidad de Huelva, angeles.delasheras@ddcc.uhu.es \\ ORCID iD: https://orcid.org/0000-0002-3640-8337 \\ Roque Jiménez Pérez \\ Universidad de Huelva, rjimenez@ddcc.uhu.es \\ ORCID iD: https://orcid.org/0000-0001-7779-0061
}

\begin{abstract}
RESUMEN: El alumnado de Formación Profesional Básica (FPB) se encuentra, con frecuencia, desmotivado ante los estudios. El alumnado de esta investigación, además, se halla en riesgo de exclusión social, caracterizándose por presentar carencias académicas, sociales, personales y emocionales. El presente trabajo, con el objetivo de alcanzar la inclusión educativa, pretende analizar el proceso de alfabetización científica de 10 de estudiantes de FPB utilizando una secuencia didáctica, cuyo diseño metodológico se basa en los principios de la educación situada. Para medir su alcance, se ha utilizado un test de contenidos adaptados que se pasa de forma previa y posterior a la secuencia y una narrativa dirigida a conocer la percepción del alumnado durante el proceso. Las respuestas son analizadas utilizando una rúbrica y una tabla de categorías. Se observa el desarrollo de aspectos que ponen en evidencia cómo la experiencia ha conseguido la inclusión educativa del alumnado.
\end{abstract}

Palabras Clave: Formación Profesional Básica (FPB), inclusión educativa, educación situada, aprendizaje significativo, alfabetización científica

Abstract: Basic Vocational Education and Training (VET) students are often unmotivated about their studies. The students in this research are also at risk of social exclusion, characterized by academic, social, personal, and emotional deficiencies. The present work, with the aim of achieving educational inclusion, aims to analyze the scientific literacy process of 10 VET students using a teaching sequence, the methodological design of which is based on the principles of situated education. To measure its scope, an adapted content test that is passed before and after the sequence and a narrative aimed at knowing the students' perception during the process has been used. Responses are analyzed using a rubric and a category table. The development of aspects that show how the experience has achieved the students' educational inclusion is observed.

KEYWORDS: Basic Vocational Education and Training (VET), educational inclusion, situated education, meaningful learning, scientific literacy

Fecha de recepción: mayo de 2021

Fecha de aceptación: octubre de 2021 


\section{INTRODUCCIÓN}

La educación es un proceso complejo basado en la interacción mutua de muchos factores, también se sabe que el fracaso escolar se debe a razones diferentes y muy versátiles (Shah, Hagell y Cheung, 2019). El fracaso escolar, o no haber completado con éxito la educación secundaria obligatoria, es un fenómeno que guarda relación con el entorno socioeconómico de los estudiantes e influye de manera decisiva en las cifras de abandono (Soler et al., 2021). Según establecen Amores y Ritacco (2015), el fracaso escolar se asocia a situaciones como: bajos rendimientos académicos, absentismo escolar, desenganche afectivo de la escuela, abandono prematuro de la educación obligatoria sin la graduación correspondiente.

En este sentido, España se coloca con la peor tasa de la Unión Europea, según datos publicados en enero de 2020 por el Ministerio de Educación y Formación Profesional (17,3\%). Además, el problema de esta tipología de alumnado se agrava por encontrarse en riesgo de exclusión social. En este sentido, se destacan algunos factores, tales como: situaciones emocionales, sociales, de salud mental, pobreza, bajo rendimiento académico, pertenecer a grupos minoritarios, malas relaciones con los docentes y/o presencia de dificultades en el hogar (Graham, White, Edwards, Potter y Street, 2019). Por ello, consideramos que, tal y como afirman Sarceda, Santos y Sanjuán (2017), los programas de FPB se presentan como una alternativa para aquellos estudiantes que han abandonado el sistema educativo, pretendiendo, entre otras cuestiones, cubrir algunas de las posibles carencias del alumnado, como pueden ser:

- Académicas: Desilusión por lo académico, evidenciado por el previo fracaso escolar (dificultades a nivel familiar, socio-ambiental, dificultades de aprendizaje no contempladas anteriormente, absentismo escolar, ...) (Puente, 2015).

- Sociales: Manifiestan dificultades diversas a nivel familiar, socio-ambiental, problemas en las relaciones sociales..., haber tenido una mala aceptación social, carencias en la resolución de conflictos en las relaciones sociales, no aceptan normas de convivencia, (Puente, 2015).

- Personales: Presentan debilidades notables en casi todos los ámbitos del desarrollo personal (Vega y Aramendi, 2010), así como dificultades en las habilidades interpersonales, problemas de autocontrol, impulsividad, dificultades de concentración, baja autoestima, autoconcepto negativo... (Puente, 2015).

- Emocionales: Alumnado caracterizado por su falta de madurez emocional (Moneo, Jiménez-Pérez y Jiménez-Palacios, 2017): conductas inadaptadas (Vega, Aramendi y Huegun, 2009 citado en García-Gómez, 2014, p. 183), conflictividad y apatía (Jiménez, 1999 citado en Amores y Ritacco, 2015, p. 209) y baja autoestima (García-Gómez, 2014), entre otras.

Estos factores de riesgo, normalmente, no se dan de manera aislada, sino que suelen presentarse asociados y de forma acumulativa (Rodríguez y Guzmán, 2019), aseverando Bisquerra (2003) que entre estas co-ocurrencias de comportamientos problemáticos destacan la violencia, la depresión, el suicidio y el consumo de drogas. En este sentido, tal y como establece López-Vélez (2018), es necesario poner atención en aquellos estudiantes en peligro de sufrir marginación, exclusión y riesgo de no alcanzar un aprendizaje con significación social. Hasta el momento, a pesar de la existencia de nuevas leyes que desde el año 2015 regulan el sistema de protección a la infancia y a la adolescencia en España, se han realizado pocas investigaciones sobre los menores en situación de riesgo (Rodríguez y Guzmán, 2019).

En nuestro caso, el objetivo de este trabajo es describir cómo la alfabetización científica que un grupo de estudiantes del segundo curso de Formación Profesional Básica puede adquirir, facilita el 
interés por la tarea y la posible inclusión educativa tras una intervención en el aula cuyas bases metodológicas se asientan en el aprendizaje de significados mediante la educación situada.

En consecuencia, nos planteamos los problemas de investigación siguientes:

El problema principal se formula según:

¿La alfabetización científica facilita la inclusión educativa de un grupo de estudiantes de FPB?

A raíz de este problema principal, surgen los siguientes subproblemas:

- ¿Es posible mejorar la alfabetización científica en un grupo de estudiantes de FPB a través del uso de una secuencia didáctica basada en la enseñanza situada?

- ¿Qué aspectos se pueden considerar básicos, en el aula, para conseguir la inclusión educativa del alumnado?

\section{FUNDAMENTOS TEÓRICOS}

\subsection{La función social de la FPB}

Las escuelas aparecen como microcosmos de la sociedad (Graham, White, Edwards, Potter y Street, 2019). Según contempla Goleman (1996), la educación completa la condición humana, preparando al individuo para la vida, posibilitando, según Bisquerra (2011), que ésta pueda evaluar de forma positiva la calidad total de su vida. El término inclusión educativa, hace referencia al derecho que toda persona tiene a una educación de calidad (González-Rojas y Triana, 2018). En este sentido, han sido numerosos y permanentes los cambios que han experimentado estos programas, dando cuenta de ello sus diferentes siglas (CIP, PGS, PIP, PCPI, ahora FPB) (Martínez-Seijo y Torrego, 2014). Así comienza el desarrollo de iniciativas, a caballo entre el sistema educativo y la educación no formal, variadas y diversas ante las diferencias territoriales autonómicas, lo que supone una implantación irregular a lo largo de todo el territorio español (Palomares y López, 2012).

Siguiendo esta estela educativa, nace la Formación Profesional Básica (en adelante FPB), que, según la LOMLOE (2020), se trata de un programa que se presenta como: "el conjunto de acciones formativas que capacitan para el desempeño cualificado de diversas formaciones" (p. 72). Este tipo de programas cumplen una función social importante (Casares, 2007) que se están abordando, con recursos limitados y con realidades de exclusión social graves (Martínez-Seijo y Torrego, 2014).

Los estudiantes logran un sentido de pertenencia a la escuela, que implica sentirse valorado como individuo sintiendo que sus necesidades son entendidas y atendidas (Graham, White, Edwards, Potter y Street, 2019), lo cual se convierte en el escenario idóneo para afrontar la problemática del alumnado objeto de estudio que, además de arrastrar situaciones graves de fracaso escolar, se encuentran en riesgo potencial de exclusión social. A raíz de esta necesidad, surge la idea de ciencia que no excluya a nadie y que esté íntimamente asociada a los principios educativos de comprensividad y equidad (Sabariego y Manzanares, 2006).

\subsection{La alfabetización científica como medida de inclusión educativa}

Una buena planificación de aula debe dirigir sus esfuerzos a ofrecer a los estudiantes igualdad de oportunidades (Sisto, Pérez-Fuentes, Gázquez y Molero 2021), considerando, en este caso, que los colectivos más frágiles requieren medidas específicas para combatir la exclusión (Afonso y Sastre, 2007). Debido a que la preocupación actual, con la necesidad de ofrecer una educación que responda a las necesidades de los estudiantes, mejorando su calidad de vida (Marín-Perabá, 2019), surge el concepto de inclusión educativa. Siguiendo lo establecido por la UNESCO (1994), se entiende que la inclusión educativa es un proceso de respuesta a la diversidad de necesidades de todos los aprendices, para no crear brechas sociales. En este sentido, es importante contemplar que entre los 
factores que obstaculizan la inclusión se encuentra el analfabetismo (Jiménez-Ramírez, 2008), convirtiéndose, como determinan Núñez y Rodríguez (2015), en un factor clave para el cambio y la justicia social y, por tanto, para la inclusión. En este sentido, se destaca que la planificación de experiencias educativas que mejoren la alfabetización científica suele estar ausente en el aula (Zimmermann, 2007).

Según Sawyer et al. (2018 citado en Shah, Hagell y Cheung, 2019, p. 10), el paso de la infancia a la edad adulta implica una amplia gama de desafíos físicos, psicológicos y sociológicos, siendo en la adolescencia, cuando aparecen problemas de salud producidos como consecuencia de los estilos de vida, como el consumo de alcohol y drogas, trastornos de comportamientos alimentarios y sexuales (Hagell, Shah y Coleman 2017). Los adolescentes van configurando sus estilos de vida en sus contextos sociales y familiares que condicionan ciertos comportamientos saludables (Camino et al., 2007), de ahí la importancia también de la competencia emocional, siendo un factor esencial para el desarrollo personal y social (Bisquerra, 2003).

La alfabetización científica va más allá del mero conocimiento del contenido científico, permitiendo que las personas se puedan involucrar críticamente en la toma de decisiones sobre temas relacionados con la ciencia (Siarova, Sternadel y Szönyi, 2019) e implicarse en discusiones públicas acerca de asuntos importantes que se relacionan con la ciencia (Gil y Vilches, 2001), logrando la satisfacción social y personal del estudiante (Merchán, 2018).

\subsection{Enseñanza situada en el diseño de la secuencia didáctica}

El hecho de que las instituciones educativas no entreguen actividades de formación adaptadas a las necesidades del estudiante, genera una necesidad de inclusión (Marín-Perabá, 2019). Para garantizar la calidad educativa, según la UNESCO (2019), hay que contar con sistemas educativos inclusivos, cuyo objetivo es responder a la diversidad de cada estudiante. Reducir la exclusión y atender a la vulnerabilidad, favorece, siguiendo a Iglesias, Madrid, Ramos, Robles y Serrano de Haro (2013), la mejora del alumnado a través de estrategias integradoras, como las establecidas por Ramírez-Valbuena (2017) de escucha, dialogo, cooperación, confianza y aceptación que posibiliten, según determina Guerrero (2012), la construcción de aprendizajes significativos y relevantes para el alumnado.

Como estrategias inclusivas, se pueden seleccionar las bases de la enseñanza situada, en la que adoptando criterios de relevancia social, se busca fortalecer la identidad (Díaz-Barriga, 2010), motivándolo y atendiendo de forma primordial al contexto y a la realidad en la que el estudiante crece y se desenvuelve (Ortega, 2017). Los contenidos deben guardar significatividad coherente, de forma que la información sea comprendida con facilidad por el alumnado (Palomino, 2018). Por otra parte, tal y como afirma Ballester (2005), los contenidos deben tener significatividad psicológica, siendo adecuados al nivel de desarrollo y conocimientos previos del estudiante, suponiendo un aprendizaje útil porque el contenido es de interés para él.

A partir de estas estrategias se logra una organización de las actividades de aprendizaje que se realizarán con los alumnos y para los alumnos con la finalidad de crear situaciones que les permitan desarrollar un aprendizaje inclusivo (Díaz-Barriga, 2003).

\section{Metodología}

\subsection{Contexto y Participantes}

El presente estudio surge en un aula de FPB de un centro educativo de la provincia de Huelva, considerado como centro de compensatoria. El alumnado que compone la comunidad del centro en el que se lleva a cabo el estudio, se caracteriza por la heterogeneidad y diversidad, en el que la implicación de las familias es prácticamente inexistente, teniendo un número significativo de menores con unas medidas temporales de acogimiento residencial. 
En concreto, los participantes en esta investigación son diez alumnos del segundo curso FPB, de la especialidad de Informática y Comunicaciones, cuyas edades oscilan entre los 16 y los 18 años, de los cuales, nueve pertenecen al género masculino y uno al femenino. Dos de los estudiantes son de etnia gitana y tres de procedencia sudamericana. El grupo comparte algunos rasgos generales, tales como acarrear un gran historial de fracaso escolar, absentismo, así como tener evidentes dificultades con las instrumentales básicas.

Nueve de ellos proceden de familias desestructuradas y siete tienen problemas con el consumo de sustancias ilegales, así como con ingesta de alcohol de forma habitual. Cuatro están diagnosticados con el Trastorno del Déficit de Atención por Hiperactividad, uno de ellos tiene Trastorno Negativista Desafiante y otro atraviesa un periodo de depresión en el momento de la investigación.

\subsection{Proceso de intervención}

La intervención se llevó a cabo a lo largo del segundo trimestre del curso académico 2018/2019, en el Módulo de Ciencias Aplicadas II. Se planifica y aplica una secuencia didáctica que fue diseñada teniendo en cuenta dos aspectos fundamentales:

1. Que los contenidos, además de encontrarse incluidos en la Orden de 8 de noviembre de 2016 de la FPB, hubieran sido pre-seleccionados por los estudiantes objeto de estudio.

2. Que atendiera a los principios metodológicos de la enseñanza situada.

Coincidiendo con los estudios de Marín-Perabá (2019), la implementación llevada a cabo en la presente investigación pretende trabajar contenidos que sean adecuados, basándonos en los intereses de los estudiantes, para facilitar el proceso de aprendizaje. Por tanto, se llevó a cabo un listado de 10 contenidos (física, medio ambiente y sanitarios) para que eligiesen en función de sus preferencias. Finalmente, seleccionaron aquellos relacionados con la salud, por este orden: Hábitos saludables, Sexualidad y Nutrición. Para esta investigación se selecciona la temática con mayor número de votos que, bajo el titulo de hábitos saludables, trata aspectos relacionados con el consumo de sustancias. En este caso, como describen Tobón, Pimienta y García (2010), en esta secuencia se trabajan problemas significativos y pertinentes para los estudiantes, convirtiendo el aula en un escenario social.

Los contenidos a tratar en esta secuencia didáctica se clasifican en tres categorías, tal y como se describe en la Tabla 1:

TABLA 1: Contenidos relativos a los Hábitos Saludables.

\begin{tabular}{lc}
\hline Categorías & Hábitos saludables \\
\hline $\begin{array}{c}\text { Adicción al } \\
\text { consumo de } \\
\text { drogas }\end{array}$ & $\begin{array}{c}\text { Descripción } \\
\text { principales características de los tipos de droga, clasificación de las drogas, } \\
\text { significado de tolerancia, dependencia y abstinencia. }\end{array}$ \\
\hline $\begin{array}{c}\text { Las drogas y } \\
\text { la salud }\end{array}$ & $\begin{array}{c}\text { Diferenciar algunos de los principales mitos y verdades sobre las drogas, } \\
\text { componentes del tabaco, consecuencias del cannabis, identificar consecuencias } \\
\text { que para la salud tiene la ingesta de alcohol. }\end{array}$ \\
\hline Tratamiento & Identificar algunas pautas a tener en cuenta en el tratamiento de un adicto. \\
\hline
\end{tabular}

Fuente: Orden 8 de noviembre de 2016 
En cuanto a la forma de implementar la secuencia en el aula, se siguen los principios metodológicos propios de la enseñanza situada, por considerarse una estrategia inclusiva, tal y como refleja la Tabla 2:

TABLA 2: Relación entre la inclusión educativa y la enseñanza situada.

\begin{tabular}{|c|c|}
\hline Inclusión educativa & Enseñanza situada \\
\hline Estudiante participativo y activo (Slee, 2019) & $\begin{array}{l}\text { Propuesta motivadora que faculta al alumnado para } \\
\text { participar activamente (Díaz-Barriga, 2003) }\end{array}$ \\
\hline $\begin{array}{l}\text { Lograr resultados académicos y sociales óptimos } \\
\text { (Slee, 2019) }\end{array}$ & $\begin{array}{l}\text { Mejora las capacidades de los estudiantes y } \\
\text { aprendizaje significativo (Díaz-Barriga, 2003) }\end{array}$ \\
\hline $\begin{array}{l}\text { Mejorar actitudes, valores de igualdad, } \\
\text { tolerancia y respeto de la diversidad (Sabando, } \\
\text { 2016). }\end{array}$ & $\begin{array}{l}\text { Desarrolla habilidades, actitudes y valores de los } \\
\text { estudiantes (Benavides, Madrigal y Quiroz, 2009). }\end{array}$ \\
\hline $\begin{array}{c}\text { Mejorar la autonomía, cooperación y cohesión } \\
\text { social (Sabando, 2016). }\end{array}$ & $\begin{array}{l}\text { Promueve la autogestión y la autonomía facilitando } \\
\text { la cohesión de grupo (Muntaner, Pinya y Mut, } \\
\text { 2020). }\end{array}$ \\
\hline $\begin{array}{l}\text { Ajustar ritmos de aprendizaje (Marín-Perabá, } \\
\text { 2019). }\end{array}$ & $\begin{array}{c}\text { Se ajusta el ritmo de aprendizaje (Benavides, } \\
\text { Madrigal y Quiroz, 2009). }\end{array}$ \\
\hline $\begin{array}{l}\text { Hacer estudio exhaustivo de cada estudiante } \\
\text { (Klinger, Mejía y Posadas, 2011), adaptando la } \\
\text { metodología: organización, procedimientos, } \\
\text { actividades, orden de contenidos y de objetivos, } \\
\text { aplicando concreción curricular (Marín-Perabá, } \\
\text { 2019). }\end{array}$ & $\begin{array}{l}\text { Metodología coherente, significativa (trascendiendo } \\
\text { lo memorístico), propositiva y de carácter social, } \\
\text { adaptándose a los estudiantes (Díaz-Barriga, 2003). }\end{array}$ \\
\hline $\begin{array}{l}\text { Implicar la afectividad (Klinger, Mejía y } \\
\text { Posadas, 2011). }\end{array}$ & $\begin{array}{l}\text { Se promueven intercambios afectivos positivos } \\
\text { (Díaz-Barriga y Hernández, 2010). }\end{array}$ \\
\hline $\begin{array}{c}\text { Aplicar normas educativas en el aula (Klinger, } \\
\text { Mejía y Posadas, 2011) }\end{array}$ & $\begin{array}{l}\text { Establecer normas de comportamiento que regulen } \\
\text { las relaciones sociales (Baquero, 2002) }\end{array}$ \\
\hline $\begin{array}{l}\text { En base a las necesidades del estudiante, se } \\
\text { seleccionan contenidos adecuados (Marín- } \\
\text { Perabá, 2019). }\end{array}$ & $\begin{array}{l}\text { Contextualización del aprendizaje (Benavides, } \\
\text { Madrigal y Quiroz, 2009), siendo adecuados al } \\
\text { nivel de desarrollo y conocimientos previos del } \\
\text { estudiante (Ballester, 2005). }\end{array}$ \\
\hline
\end{tabular}

Fuente: Elaboración propia

La secuencia didáctica se divide en tres fases y en cada una de ellas se programa un tipo de actividades, descritas por Díaz-Barriga y Hernández (2010), que pretenden promover aprendizajes significativos, implementando, como establece Sagástegui (2004), procesos cognoscitivos que impliquen percepciones, significados, intenciones, interacciones y recursos. En la Tabla 3, se muestran las fases, el tipo de actividad y la descripción correspondiente. 
TABLA 3. Fases y sesiones de las secuencias didácticas.

\begin{tabular}{|c|c|c|}
\hline $\begin{array}{l}\text { Fases de la } \\
\text { enseñanza } \\
\text { situada }\end{array}$ & $\begin{array}{l}\text { Tipo de actividad } \\
\text { (Díaz-Barriga y } \\
\text { Hernández, 2010) }\end{array}$ & Descripción de la actividad \\
\hline Fase apertura & $\begin{array}{l}\text { Discurso docente y } \\
\text { discusiones guiadas } \\
\text { (Sesión } 1)\end{array}$ & $\begin{array}{l}\text { Introducción a los Hábitos Saludables. } \\
\text { Se pregunta al alumnado acerca de su experiencia con las } \\
\text { drogas y sus hábitos de consumo. }\end{array}$ \\
\hline \multirow[t]{5}{*}{$\begin{array}{c}\text { Fase } \\
\text { desarrollo }\end{array}$} & $\begin{array}{l}\text { Discurso docente e } \\
\text { ilustraciones (Sesión } \\
\text { 2) }\end{array}$ & $\begin{array}{l}\text { Explicación sobre las adicciones, clasificación y sus } \\
\text { principales conceptos asociados. Organización de los } \\
\text { grupos para la actividad. }\end{array}$ \\
\hline & $\begin{array}{l}\text { Aprendizaje basado en } \\
\text { el análisis y discusión } \\
\text { de casos (ABADC), } \\
\text { Aprendizaje en equipo } \\
\text { e Investigación en } \\
\text { grupo (Sesiones } 3 \text { y 4) }\end{array}$ & $\begin{array}{l}\text { Propuesta de caso práctico de drogas para cada grupo: } \\
\text { tienen que indagar, definir y buscar solución adoptando } \\
\text { el rol de experto. }\end{array}$ \\
\hline & $\begin{array}{l}\text { Rompecabezas (Sesión } \\
5 \text { ) }\end{array}$ & $\begin{array}{c}\text { Planteamiento de soluciones al resto de casos, analizando } \\
\text { en cada caso, sus consecuencias, tratamientos y } \\
\text { soluciones. }\end{array}$ \\
\hline & $\begin{array}{l}\text { Discurso docente y } \\
\text { Controversia } \\
\text { académica (Sesiones } 6 \\
\text { y 7) }\end{array}$ & $\begin{array}{c}\text { Se explica la prevención y tratamiento de las adicciones. } \\
\text { Se divide la clase en dos para que argumenten la defensa } \\
\text { a favor o en contra de la legalización de las drogas. } \\
\text { Puesta en común. Debate. }\end{array}$ \\
\hline & $\begin{array}{c}\text { Discurso docente } \\
\text { Imágenes (Sesión 8) }\end{array}$ & $\begin{array}{l}\text { Debate sobre personas famosas que han superado la } \\
\text { adicción a las drogas. Se introducen imágenes y videos. }\end{array}$ \\
\hline Fase cierre & (ABADC) (Sesión 9) & $\begin{array}{l}\text { Recapitulación y reorganización de sesiones previas, } \\
\text { individualizando lo estudiado. }\end{array}$ \\
\hline
\end{tabular}

Fuente: Elaboración propia

\subsection{Instrumentos para la investigación}

A continuación, se describen los instrumentos empleados durante la investigación, tanto los de primer orden o de recogida de información como los de segundo orden o de análisis.

\section{Instrumentos de recogida de información}

Test de contenidos adaptados: Para dar respuesta al primero de los subproblemas, a partir de los contenidos concretados en la Tabla 1, se diseñó un test de contenidos adaptados de elaboración propia, compuesto por 8 ítems, combinándose preguntas de desarrollo, cerradas o de opción de múltiple. Para comprobar la idoneidad de este cuestionario, se realizó un doble proceso de validación: por un lado, es analizado por un grupo de expertos y, por otro, se realiza una prueba piloto con un grupo de 15 alumnos de $3^{\circ}$ y $4^{\circ}$ de la ESO del mismo centro. Los resultados obtenidos aconsejaron suprimir las preguntas abiertas o reflexivas por su complejidad para los estudiantes, por lo que se elaboró un segundo test de preguntas cerradas y de opción múltiple, que se volvió a pasar a un grupo 
de diez estudiantes del primer curso de la FPB y, debido a la conformidad de éstos respecto al entendimiento del cuestionario, se tomó como definitivo, quedando, de forma esquemática, tal y como se refleja en la Tabla 4:

TABLA 4: Cuestionario de "Hábitos Saludables"

1. Con respecto al consumo de sustancias, indica verdadero o falso: "El tabaco no relaja, sino que estimula el sistema nervioso/El alcohol disminuye tus reflejos, te hace más lento/..."

2. Indica cuáles de las drogas de las imágenes son estimulantes, depresoras o alucinógenas:

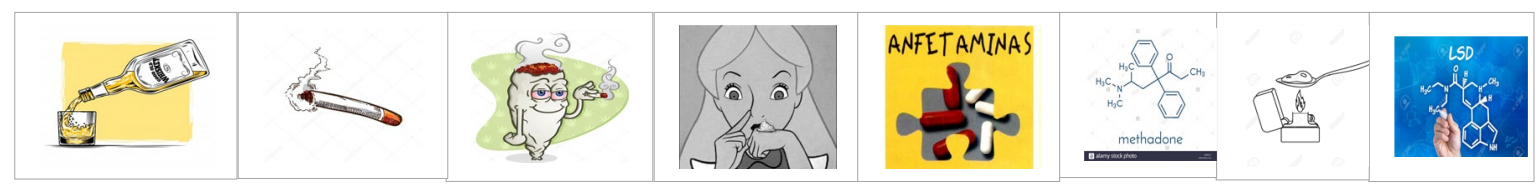

3. Identifica las frases correctas: "si tú controlas, las drogas no tienen por qué engancharte; todas las drogas alteran el sistema nervioso; ...

4. Cuando tenemos un familiar o amigo que tiene un problema de drogadicción es importante ayudarle: indica si las siguientes pautas de tratamiento son correctas: Existe un único tratamiento para todos los adictos; cada tratamiento debe adaptarse a las circunstancias personales...

5. Marca con una "X" los componentes que piensas que lleva el tabaco: nicotina, acetona, metanol, carburante para cohetes, ...

6. Indica con una " $X$ " qué consecuencias de las que se exponen a continuación, están asociadas al consumo de cannabis: debilitación de los huesos, debilitación de los dientes, ...

7. Relaciona cada término (tolerancia, dependencia, síndrome de abstinencia) con su definición correspondiente.

8. Indica cuál de las siguientes complicaciones para la salud son consecuencias de la ingesta de alcohol: ahogamiento con tu propio vómito, paro cardíaco, irregulares latidos del corazón ...

Fuente: Elaboración propia

Este cuestionario se aplicó a estudiantes del segundo curso de la FPB antes y después de la secuencia didáctica.

Narrativas: Para dar respuesta al segundo subproblema, se solicita a los estudiantes la elaboración de una narrativa sobre su experiencia vivida durante el proceso de enseñanza-aprendizaje. Para dirigir su atención hacia nuestros intereses y, debido también a las características del alumnado, la narrativa se presenta en forma de preguntas, en concreto 16 preguntas. En la Tabla 5, se exponen algunas de las principales estrategias educativas inclusivas que, a través de la educación situada, se adoptan en esta investigación y la relación que éstas tienen con el análisis de las narrativas de los estudiantes a través de la tabla de categorías. 
TABLA 5. Relación metodología inclusiva con la tabla de categorías de la narrativa.

Estrategias educativas aplicadas
Relación con la narrativa según la tabla de categorías / Preguntas

(P) de la Narrativa

\begin{tabular}{|c|c|}
\hline $\begin{array}{c}\text { Ajustar ritmos de } \\
\text { aprendizaje (Marín-Perabá, } \\
\text { 2019). }\end{array}$ & $\begin{array}{l}\text { Papel docente (organización del tiempo). } \\
\text { P14: ¿Os deja tiempo suficiente para terminar las actividades en } \\
\text { clase? ¿Es flexible con los plazos de entrega? }\end{array}$ \\
\hline \multirow{7}{*}{$\begin{array}{l}\text { Tras hacer un estudio } \\
\text { exhaustivo de cada } \\
\text { estudiante, adaptar la } \\
\text { metodología (Marín- } \\
\text { Perabá, 2019). }\end{array}$} & Papel docente (Organización de contenidos y de actividades). \\
\hline & $\begin{array}{l}\text { P1: La metodología entre el primer y el segundo trimestre ha sido } \\
\text { diferente, ¿has notado algún cambio? ¿cuál? }\end{array}$ \\
\hline & P2: ¿Cómo te ha gustado más trabajar? ¿Te has aburrido? \\
\hline & P3: ¿Qué es lo que te ha motivado para trabajar? \\
\hline & $\begin{array}{l}\text { P4: ¿Se hacen siempre las mismas cosas o van cambiando? ¿Por } \\
\text { qué? }\end{array}$ \\
\hline & $\begin{array}{c}\text { P5: De todas las actividades que has hecho a lo largo del segundo } \\
\text { trimestre, ¿cuál te ha gustado más? ¿por qué? }\end{array}$ \\
\hline & $\begin{array}{l}\text { P6: ¿A lo largo de este trimestre te has divertido en las clases de } \\
\text { ciencias? }\end{array}$ \\
\hline \multirow{3}{*}{$\begin{array}{l}\text { Papel pertinente del } \\
\text { estudiante, para que sean } \\
\text { sujetos activos (Castillo, } \\
\text { 2016). }\end{array}$} & Papel estudiante (Cómo trabaja en el aula). \\
\hline & $\begin{array}{l}\text { P 10: Generalmente, ¿Sueles trabajar de forma activa durante el } \\
\text { transcurso de las clases? }\end{array}$ \\
\hline & $\begin{array}{c}\text { P 16: ¿Dais vuestra opinión en cuanto a cualquier tema } \\
\text { relacionado con la clase? ¿La profesora tiene en cuenta vuestras } \\
\text { opiniones? }\end{array}$ \\
\hline \multirow{3}{*}{$\begin{array}{l}\text { Implicar la afectividad } \\
\text { (Klinger, Mejía y Posadas, } \\
\text { 2011). }\end{array}$} & $\begin{array}{c}\text { Interrelación estudiante-docente (Cómo interrelaciona con el } \\
\text { alumnado). }\end{array}$ \\
\hline & $\begin{array}{l}\text { P 12: ¿Cómo resuelve la profesora los conflictos surgidos en el } \\
\text { aula? }\end{array}$ \\
\hline & $\begin{array}{l}\text { P 15: ¿Cuándo la profesora está en clase, el ambiente es bueno? } \\
\text { ¿Es mejor que con otros profesores, igual o peor? ¿Por qué? }\end{array}$ \\
\hline \multirow{4}{*}{$\begin{array}{c}\text { En base a los intereses y } \\
\text { necesidades del estudiante, } \\
\text { se seleccionan contenidos } \\
\text { adecuados (Marín-Perabá, } \\
\text { 2019). }\end{array}$} & $\begin{array}{l}\text { Papel de las ciencias experimentales (Relevancia de contenidos). } \\
\qquad 8 \text { y } 9 .\end{array}$ \\
\hline & $\begin{array}{l}\text { P 7: ¿Has aprendido cosas nuevas? ¿Se pueden aplicar en tu vida } \\
\text { o te parecen cosas inútiles? }\end{array}$ \\
\hline & $\begin{array}{c}\text { P 8: ¿Qué os ha gustado más? ¿La nutrición? ¿Los hábitos } \\
\text { saludables? O ¿la sexualidad? ¿por qué? ¿Cuál de los tres temas } \\
\text { os parece más interesante para la vida? }\end{array}$ \\
\hline & $\begin{array}{c}\text { P 9: Alguno de los tres temas anteriores, ¿te han parecido } \\
\text { aburridos? ¿Cuál? ¿Por qué? }\end{array}$ \\
\hline
\end{tabular}

Fuente: Elaboración propia 
Instrumentos de análisis de datos

Rúbrica: Para evaluar los resultados obtenidos en los test de contenidos (Anexo 1), se analizan las respuestas a todas las preguntas en cada uno de los estudiantes, asignándole un valor de 3, 2 y 1 a cada respuesta, en función del nivel en el que se encuentra: deseable, medio y basal, respectivamente.

Tabla de categorías: Para evaluar los resultados obtenidos en las narrativas (Anexo 2), analizándose, por cada estudiante, cada una de las respuestas dadas, asignándole en cada caso el valor de 3,2 y 1 en función de que se encuentra en la categoría: deseable, buena e insuficiente, respectivamente.

\section{RESUltados Y ANÁlisis de dATOS}

Se presentan a continuación, los resultados obtenidos en los test de contenidos adaptados de forma previa y posterior a la aplicación de la secuencia didáctica (Gráfico 1).

GRÁFICO 1: Análisis de los resultados de los test de contenidos adaptados previos y posteriores a la aplicación de la secuencia didáctica.

\section{Hábitos de vida saludables}

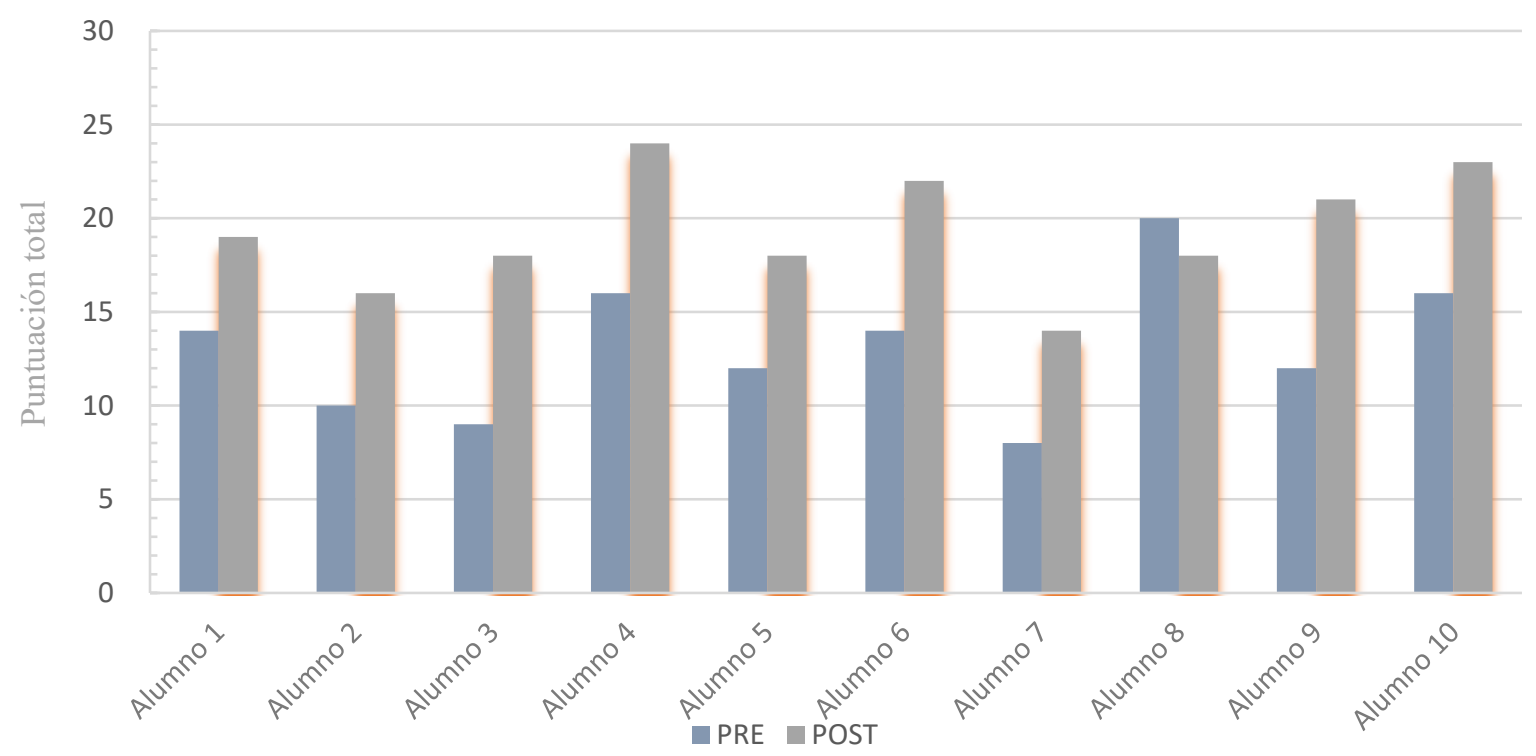

Fuente: Elaboración propia

Al analizar los test de contenidos, obtenemos el gráfico de la Figura 1, que muestra que tras la secuencia se produce una mejora en la adquisición de contenidos en nueve de los diez estudiantes, teniendo en consideración que el único alumno que no mejora los resultados atravesaba un episodio depresivo y se encontraba en tratamiento psiquiátrico en el momento de la intervención.

Realizamos la categorización de las narrativas, ayudándonos para ello de la tabla de categorías (Anexo 2). Los resultados obtenidos se reflejan en la Tabla 6, en la que se excluye a los alumnos A3 y A7 por negarse a participar, debido a un agravamiento en el consumo de sustancias tóxicas. 
TABLA 6. Resultados de la categorización de las narrativas

\begin{tabular}{cccccccccc}
\hline Categoría & Subcategoría & \multicolumn{7}{c}{ Alumnado } \\
\hline & Organización del tiempo & 3 & 3 & 3 & 3 & 3 & 3 & 3 & 3 \\
\hline Papel docente & Organización de contenidos & 3 & 3 & 3 & 3 & 3 & 3 & 3 & 3 \\
\hline & Organización de actividades & 3 & 3 & 3 & 3 & 3 & 3 & 3 & 3 \\
\hline $\begin{array}{c}\text { Papel del alumnado } \\
\text { Interacción estudiante- } \\
\text { docente }\end{array}$ & Cómo trabaja en el aula & 3 & 3 & 3 & 3 & 3 & 2 & 3 & 3 \\
\hline Papel de las CCEE & alnteracciona con el & 3 & 3 & 3 & 3 & 3 & 3 & 3 & 3 \\
\hline
\end{tabular}

Fuente: Elaboración propia

Coincidiendo con los estudios de Sisto, Pérez-Fuentes, Gázquez y Molero (2021), desde esta investigación se entiende que el modelo educativo basado en la inclusión educativa debe adaptarse al estudiante y sus necesidades. En este sentido, los estudiantes, mayoritariamente, en sus narrativas afirman que los contenidos les resultaron interesantes y útiles, sintiéndose motivados logrando no aburrirse, coincidiendo en esta aseveración con Ayllón, Gómez y Rodríguez (2013), en que adaptar la metodología a las necesidades de los estudiantes resulta básico para lograr la inclusión educativa y con Marín-Perabá (2019), en la importancia que tiene para este fin, la selección de los contenidos. Además, las narrativas reflejan que los estudiantes admiten haber adoptado un rol activo y participativo en el aula, entendiendo, como contemplan los estudios de Slee (2019), que este aspecto es fundamental para mejorar la inclusión educativa. Finalmente, los estudiantes afirman haber implicado afectos, por lo que, tal y como establece Kingle, Mejía y Posadas (2011), no se puede entender la inclusión educativa sin que en el proceso se refleje el aspecto afectivo.

Tal y como refleja la tabla 6 , en cuanto al papel docente y sus subcategorías, todos los estudiantes participantes se ubican en la categoría deseable. En cuanto a la organización del tiempo opinan que los ritmos de aprendizajes son adecuados y que se ajustan a sus necesidades. Por ejemplo, el alumno A10 afirma que "deja mucho tiempo siempre porque ella sabe que sin presiones terminamos mejor".

En cuanto a la organización de contenidos, encontramos que todos los estudiantes detectan un cambio en positivo de la metodología con respecto al trimestre anterior a la intervención, sintiendo que esta nueva forma de trabajo les resulta más atractiva y motivadora. Por ejemplo, el alumno A1, afirma que "el trabajo ha sido más entretenido porque los temas los escogimos nosotros y son más interesantes que el cambio climático o la tabla periódica" y A2 "en este ha sido mejor porque hemos hablado de cosas más interesantes y parece que tengo a la profesora hablándome todo el día. Hemos elegido los temas que íbamos a estudiar y eso mola".

Todos, además afirman no haberse aburrido y haberse sentido motivados para trabajar en clase. El alumno A6 afirma "el trabajo no es aburrido, además la maestra motiva mucho porque no se desespera eso si, si nos peleamos se pone seria tela", también A10 considera "mi profe que es la animadora como yo la llamo, está siempre diciéndonos cosas para que hagamos las tareas y se inventa juegos y formas de estudiar raras pero chulas, al final te hartas de trabajar y casi no te enteras" o A2 "la profesora te anima mucho y es paciente, eso te hace estar más relajado y si te equivocas, pues tampoco suspendes". Todos los estudiantes manifiestan haberse divertido durante el trascurso de las clases. El alumno A6 afirma "molan las clases porque al final te diviertes", A8 "aunque a veces nos hemos enfadado entre nosotros, también nos hemos divertido y nos hemos reído mucho". En esta 
línea, todos también manifiestan que las clases no eran monótonas, por lo que A9 dice que "los trabajos han sido diferentes en el segundo trimestre y si no hubiera sido así yo habría pasado de hacer nada de nada."

Además, todo el alumnado coincide en que han podido divertirse en clase mientras trabajaban, por ejemplo, el alumno A4 dice que "lo raro es no divertirse, aunque no se para de trabajar", también A10 va en ese sentido "me parecen clases divertidas porque ella nos deja trabajar a nuestro rollo y siempre hacemos cosas que nos gustan, aunque estudiar es duro".

En cuanto al papel de alumnado, tal y como refleja la Tabla 6, todos los estudiantes participantes se ubican en la categoría deseable, salvo el alumno A8 que se encuentra en la categoría intermedia, aunque llega a decir que "nos tiene en cuenta para todo". El resto reconoce haber trabajado de forma activa y dinámica en el aula y haberse sentido libres para opinar en clase. Por ejemplo, el alumno A9 "Me cuesta horrores trabajar, pero parece que ha costado menos", mientras que A10 "Generalmente me cuesta mucho centrarme porque tengo problemas en casa, pero me he conseguido olvidar", A4 "Yo trabajo a tope en todas las clases porque me gusta trabajar relajado" o A5 "Nunca me ha dado por trabajar así, volaba el tiempo". A2 "siempre nos pregunta las cosas que no nos gustan y tenemos confianza para hablar con ella" o A10 "yo siempre digo lo que pienso, sin insultar y pegarle a nadie".

En lo que respecta a la interrelación estudiante-docente, todos los estudiantes se ubican en la categoría deseable, según refleja la Tabla 6. En su conjunto, todos los estudiantes afirman haber percibido un buen clima de aula con una aportación positiva de la docente. El alumno A3 "el ambiente con ella es bueno porque nos calma cuando no estamos bien", A9 "con la docente el ambiente siempre se suaviza, la maestra nunca te deja tirado" o A10 "Es mejor porque escucha y nos entiende a los estudiantes y cuando estamos en clase el trabajo es más relajado y más entretenido". Por otra parte, todos los estudiantes participantes consideran que la docente media en los conflictos a través del diálogo y manteniendo las normas. El alumno A6 "los conflictos ella los soluciona hablando y con calma".

Finalmente, en cuanto al papel de las ciencias experimentales, tenemos según lo que refleja la Tabla 6, que, salvo el alumno A8 que se encuentra en la intermedia, el resto de participantes se ubican en la categoría deseable. De forma general, manifiestan haber tenido interés por los contenidos trabajados a lo largo de la intervención y haber encontrado aplicación para su vida cotidiana. El alumno A1 afirma que "todos los contenidos son interesantes para la vida porque no veas si comemos mierdas, pero vamos lo mejor ha sido el tema del tabaco el alcohol y la droga y la droga también en el embarazo", mientras que A5 "el tema de la droga ha sido un descubrimiento en muchas cosas aunque yo también he enseñado cosas a la docente que no sabía", A6 "el tema de las drogas porque yo pensaba que lo sabía todo pero para nada", y A8 "con el tema de los porros he flipado un poco porque fumo todos los días y ahora me da un poco de cosa." En lo que se refiere a cómo se han sentido al comprobar que estaban aprendiendo ciencias, todos afirman que el aprender les ha motivado a seguir trabajando y estudiando. El alumno Al afirma que "puede ser la primera vez que ves los resultados de los estudios y ya no puedes parar, te enganchas", mientras que A2 "es la primera vez que saco un 10 y eso te motiva", A4 "te enteras y eso en ciencias no es lo normal porque las ciencias son para los empollones y punto" o A9 "ver que te salen las cosas te hace feliz y quieres más y faltas menos"

En general, manifiestan de forma conjunta su motivación, activando los distintos aspectos de cómo valoran la tarea en cuanto a utilidad, importancia e interés, junto al valor del aspecto afectivo tan necesario en su desarrollo personal. Lo que podemos resumir en lo que expresa A1 "no he faltado los miércoles si quiera para entregar las tareas" y A6 "nunca me he sentido tan valorado por un maestro." 


\section{REFLEXIONES FINALES}

A lo largo de este trabajo hemos analizado si es posible alfabetizar científicamente a un alumnado de FPB a través de un cambio metodológico. Los resultados obtenidos según el test de contenidos adaptados, reflejan que en general, evolucionan de forma positiva, lo que supone una mejora en la tendencia para aprender contenidos científicos en la mayor parte del grupo de estudiantes participante.

En este sentido, en la línea de Greca y Jerez (2017), desde esta investigación se entiende que el aprendizaje de tipo constructivista mejora la alfabetización científica de los estudiantes vulnerables, por lo que estos resultados parecen indicar que la propuesta planteada, una secuencia didáctica diseñada bajo los principios de la educación situada, que a su vez sigue las bases de la inclusión educativa, es viable para trabajar contenidos científicos en pro de la alfabetización.

En este sentido, de acuerdo con las respuestas analizadas en las narrativas, parece que la metodología usada, se ha ajustado adecuadamente a algunos de los principios fundamentales de la inclusión educativa. Los estudiantes reflejan haber tenido un papel participativo durante el proceso, coincidiendo con los estudios de Sisto, Pérez-Fuentes, Gázquez y Molero (2021), en cómo este aspecto es muy relevante para alcanzar la inclusión educativa. Además, afirman que los contenidos fueron motivadores e inspiradores para ellos, habiendo tenido muy presente la parte afectiva a lo largo de todo el proceso.

Este conjunto de factores que se interrelacionan entre sí íntimamente, facilita la inclusión educativa, además de la alfabetización científica de los estudiantes.

\section{Referencias}

Ayllón, M., Gómez, I. y Rodríguez, L. (2013). La inclusión: un camino en construcción. Revista científica electrónica de Educación y Comunicación en la Sociedad del Conocimiento, 13(2). DOI: $10.30827 /$ eticanet.v13i2.11997

Afonso A. y Sastre S. (2017). La exclusión social en España: Factores, colectivos en riesgo y papel de los bancos de alimentos. Cátedra Banco de Alimentos de la UPM. Recuperado de https://www.bancodealimentos.es/wp-content/uploads/2017/10/Exclusion-social.pdf

Amores F. J. y Ritacco M. (2015). Programas de Cualificación Profesional Inicial: Percepciones y Perspectivas de Futuro de los Estudiantes en la Provincia de Granada. Revista Internacional de educación para la Justicia Social 4(2), 201-218. DOI:15366/riejs2015.4.2.

Baquero R. (2002). Del experimento escolar a la experiencia educativa. La transmisión educativa desde una perspectiva psicológica situacional. Perfiles Educativos, XXIV (98), 57-75.

Ballester A. (2005). El aprendizaje significativo en la práctica. Equipos de investigación y ejemplos en didáctica de la geografía. Congreso Internacional Virtual de Educación. Escola de Formació en Mitjans Didàctics de la Universitat de las Islas Baleares. Palma de Mallorca, España.

Benavides, D., Madrigal, V.M. y Quiroz, A.P. (2009). La enseñanza situada como herramienta para el logro de un aprendizaje significativo. Centro de Documentación sobre Educación, septiembre.

Bisquerra R. (2003). Educación emocional y competencias básicas para la vida. Revista de Investigación Educativa, 21(1), 7-43.

Bisquerra R. (2011). Educación emocional. Propuestas para educadores y familias. Bilbao: Desclée de Brouwer.

Camino H., Irujo A., Gastón J.M., Escribano L., Corretgé M.T., García M.A., Castiella F., Mateo M.V., García J.L., Elizalde L. y Echauri M. (2007). Aulas saludables, adolescentes competentes. Navarra: Gobierno de Navarra. Recuperado de 
http://www.navarra.es/NR/rdonlyres/5209B83F-9C8D-42F1-B7EB-

B542FA45D483/0/Aulassaludablescast.pdf

Castillo J.R. (2016). Docente inclusivo, aula inclusiva. Revista nacional e internacional de educación inclusiva, 9(2), 264-275.

Casares, M.E. (2007). Estudio descriptivo de los programas de garantía social de iniciación profesional en los centros de Granada. [Tesis doctoral, Universidad de Granada]

Díaz-Barriga F. (2003). Cognición situada y estrategias para el aprendizaje significativo. Revista Electrónica de Investigación Educativa, 5(2), 105-117

Díaz-Barriga F. y Hernández G. (2010). Estrategias docentes para un aprendizaje significativo. Una interpretación constructivista. México DF: McGraw-Hill.

García-Gómez S. (2014). La investigación sobre el alumnado de los Programas de Cualificación Profesional Inicial ¿Punto de partida de la Formación Profesional Básica? Revista de Investigación en Educación, 12(2), 176-190.

Gil D. y Vilches A. (2001). Una alfabetización científica para el siglo XXI: Obstáculos y propuestas de actuación. Revista Investigación en la Escuela, 43, 27-37.

Goleman D. (1996). Inteligencia Emocional. Barcelona: Kairós.

González-Rojas Y. y Triana D. A. (2018). Actitudes de los docentes frente a la inclusión de estudiantes con necesidades educativas especiales. Educación y Educadores, 21(2), 200-218. DOI:10.5294/edu.2018.21.2.2

Graham B., White C., Edwards A., Potter S. \& Street C. (2019). School exclusion: a literature review on the continued disproportionate exclusion of certain children. London: Department for Education of United Kingdom. Recuperado de https://assets.publishing.service.gov.uk/government/uploads/system/uploads/attachment_data/ file/800028/Timpson_review_of_school_exclusion_literature_review.pdf

Greca I. M. y Jerez E. (2017). Propuesta para la enseñanza de Ciencias Naturales en Educación Primaria en un aula inclusiva. Revista Eureka sobre Enseñanza y Divulgación de las Ciencias, 14(2), 385-397. DOI: 10.25267/Rev_Eureka_ensen_divulg_cienc.2017.v14.i2.07

Guerrero C. (2012). Hacia la construcción de procesos y prácticas "exclusivas": metodologías para la intervención. Quadernsanimacio, 16(3), 1-16.

Hagell A., Shah R \& Coleman, J. (2017). Key data on young people 2017. London: The Association for Young People's Health (AYPH). Recuperado de http://www.ayph.org.uk/keydata2017/FullVersion2017

Iglesias B., Madrid L., Ramos C., Robles y Serrano de Haro A. (2013). Metodologías innovadoras e inclusivas en educación secundaria: los grupos interactivos y la asamblea de aula. Tendencias pedagógicas 21,63-78.

Jiménez-Ramírez M. (2008). Aproximación teórica de la exclusión social: complejidad e imprecisión del término. Consecuencias para el ámbito educativo. Revista Estudios Pedagógicos XXXIV 1, 173-186. DOI:10.4067/S0718-07052008000100010

Kingle C.L., Mejía C.M. y Posada L.M (2011). La inclusión educativa: un escenario de expresiones afectivas como mediadoras del aprendizaje. Plumilla Educativa 8(2), 176-190. DOI: 10.30554/plumillaedu.8.491.2011

LOMLOE (2020). Ministerio de Educación y Formación Profesional. Ley Orgánica 3/2020, de 29 de diciembre. Recuperado de: https://www.educacionyfp.gob.es/dam/jcr:f183be05-117d-4806b24a-2f9f52d9b37f/loe-con-lomloe-texto.pdf.

López-Vélez A. L. (2018). Escuela inclusiva. El derecho a la equidad y la excelencia educativa. Bilbao: Servicio Editorial de la Universidad del País Vasco.

Marín-Perabá C. (2019). Enfoques educativos de la concepción de integración e inclusión. Revista Internacional de apoyo a la inclusión, logopedia, sociedad y multiculturalidad. 5(1),115-124. https://doi.org/10.17561/riai.v5.n1 
Martínez-Seijo M. L. y Torrego J. C. (2014). Programas y medidas educativas en España para alcanzar objetivos europeos. Revista Electrónica de Investigación Educativa 16(2), 119-134. Recuperado de http://redie.uabc.mx/vol16no2/contenido-mtnez- torrego.html

Merchán A. (2018). Propuesta para promover la alfabetización científica en alumnos de $2^{\circ}$ de la ESO mediante actividades Ciencia-Tecnología-Sociedad [Trabajo Final de Máster, Universidad Internacional de la Rioja]

Moneo A., Jiménez- Pérez R. y Jiménez-Palacios R. (2017). Evolución de la madurez emocional en alumnos de Formación Profesional Básica mediante el empleo de actividades fuera del contexto escolar en el área de las ciencias naturales. Revista Eureka sobre Enseñanza y Divulgación de las Ciencias 14(1), 69-85. Recuperado de https://revistas.uca.es/pre/index.php/eureka/article/view/3006

Muntaner, JJ. Pinya, C. y Mut, B. (2020). El impacto de las metodologías activas en los resultados académicos: Un estudio de casos. Revista de currículum y formación del profesorado, 24 (1), 97-114. DOI: 10.30827 /profesorado.v2i1.8846

Núñez J.A. y Rodríguez M.S. (2015). El desafío de alfabetizar en el siglo XXI. Dimensiones y propuestas en torno a la alfabetización. Verbeia. Revista de estudios filológicos 0, 139-157.

Ortega F. (2017) Principios e implicaciones del Nuevo Modelo Educativo. Revista latinoamericana de estudios educativos 47(1), 43-62. DOI: 10.48102/rlee.2017.47.1.157

Palomares A. y López-Sánchez S. (2012). La respuesta a la diversidad de los programas de garantía social hacia los programas de cualificación profesional inicial. Revista Española de Educación Comparada 20, 249-274. DOI:10.5944/reec.20.2012.7600

Palomino J. O. (2018). Aprendizaje significativo y las actitudes hacia las matemáticas en estudiantes del VII ciclo, en la Institución Educativa 1227-Ate 2018. [Tesis de grado, Escuela de posgrado Universidad César Vallejo, Lima]

Puente J. M. (2015). Una visión de la Formación Profesional Básica y de la utilidad de la tutoría en estas enseñanzas. [Trabajo fin de máster, Universidad de Cantabria]

Ramírez-Valbuena, W.A. (2017) La inclusión: una historia de exclusión en el proceso de enseñanzaaprendizaje. Cuadernos de Lingüistica Hispánica 30, 211-230. DOI:10.19053/0121053X.n30.0.6195

Rodríguez D. y Guzmán R. (2019). Rendimiento académico de adolescentes declarados en situación de riesgo. Revista de Investigación Educativa 37(1), 147-162. DOI: 10.6018/rie.37.1.303391

Sabando D.S. (2016). Inclusión educativa y rendimiento académico. Relación entre el grado de Inclusión y el Rendimiento académico en las Escuelas Públicas de Primaria de Cataluña [Tesis doctoral, Universidad de Barcelona]

Sabariego J.M. y Manzanares M. (2006, 19-23 de junio). Alfabetización científica [ponencia]. I Congreso iberoamericano de ciencia, tecnología, sociedad e Innovación CTS+I. Ciudad de México. Recuperado de https://www.oei.es/historico/noticias/spip.php?article820

Sagástegui D. (2004). Una apuesta por la cultura: el aprendizaje situado. Revista Electrónica Sinéctica 24, 30-39.

Sarceda, M.C., Santos-González, M.C. y Sanjuán, M.M. (2017). La formación profesional básica: ¿alternativa al fracaso escolar? Revista de Educación, 378, 78-102. DOI 10.4438/1988-592XRE-2017-378-362

Shah R., Hagell A. \& Cheung R. (2019). International comparisons of health and wellbeing in adolescence and early adulthood. London: UK. Association For Young People Health.

Siarova H., Sternadel D. \& Szönyi E. (2019). Research for CULT Committee-Science and Scientific Literacy as an Educational Challenge. European Parliament. Recuperado de http://bit.ly/2TCc6Uy

Sisto M., Pérez-Fuentes, M.C., Gázquez-Linares, J.J. \& Molero-Jurado, M.M. (2021). Actitudes hacia la inclusión educativa de alumnos con discapacidad: variables relativas al profesorado y 
a la organización escolar en Educación Primaria. Revista Electrónica Interuniversitaria de Formación del Profesorado, 24 (1), 221-237. DOI: 10.6018/reifop.397841

Slee, R. (2019). Defining the scope of inclusive education. Think piece prepared for the 2020 Global Education Monitoring Report Inclusion and education. United Nation Educational, Scientific and Cultural Organization (UNESCO)

Soler A., Martínez-Pastor J.I., López-Meseguer R., Valdés M.T., Sancho M.A., Morillo B. y Cendra L. (2021). Mapa del abandono educativo temprano en España. Madrid: Fundación Europea Sociedad y Educación.

Tobón S., Pimienta J. y García J. (2010) Secuencias Didácticas: aprendizaje y evaluación de competencias. México D.F: Pearson-Prentice Hall.

UNESCO. (1994). Una revisión de las actividades de la UNESCO a la luz de la Declaración de Salamanca (Conferencia Mundial sobre necesidades educativas especiales: acceso y calidad). Ministerio de educación y ciencia de España. Recuperado de https://sede.educacion.gob.es/publiventa/descarga.action?f_codigo_agc=1597_19

UNESCO. (2019). Todas y todos los estudiantes cuentan. (Foro internacional sobre inclusión y equidad en la educación). Recuperado de https://en.unesco.org/sites/default/files/2019-foruminclusion-concept-note-es.pdf

Vega A. y Aramendi P. (2010). Entre el fracaso y la esperanza. Necesidades formativas del alumnado de los programas de cualificación profesional inicial. Educación XXI, 13(1), 39-63. DOI: 10.5944/educxx1.13.1.276

Zimmerman C. (2007). 'The development of scientific thinking skills in elementary and middle school'. Developmental Review, 27(2), 172- 223. DOI: 10.1016/j.dr.2006.12.001

\section{CÓMO CITAR ESTE ARTÍCULO}

López Santos, S., De las Heras Pérez, M.A. y Jiménez Pérez, R. (2021). La alfabetización científica en estudiantes de Formación Profesional Básica a través de una secuencia adaptada hacia la inclusión educativa. Didáctica de las ciencias experimentales y sociales, 41, 137-154. DOI: 10.7203/DCES.41.20872 


\section{AnEXOS}

ANEXO 1. Rúbrica de los Hábitos Saludables.

Rúbrica Hábitos Saludables

\begin{tabular}{|c|c|c|c|c|}
\hline \multicolumn{5}{|c|}{ Rúbrica Hábitos Saludables } \\
\hline & & \multicolumn{3}{|c|}{ Niveles de progresión } \\
\hline Categorías & Descripción & BASAL & MEDIO & $\begin{array}{c}\text { DESEAB } \\
\text { LE }\end{array}$ \\
\hline \multirow[t]{3}{*}{ Las adicciones } & $\begin{array}{l}\text { Definición. Identificar las frases correctas relativas } \\
\text { al concepto de droga, su relación con el sistema } \\
\text { nervioso, el control, la dependencia, su consumo en } \\
\text { relación a factores externos como la juventud o la } \\
\text { pobreza del consumidor o las principales } \\
\text { características de los tipos de droga. }\end{array}$ & $\begin{array}{l}\text { Identifica } \\
\text { entre } 0 \text { y } 4\end{array}$ & $\begin{array}{l}\text { Identifica } \\
\text { entre } 5 \text { y } 9\end{array}$ & $\begin{array}{l}\text { Identifica } \\
\text { entre } 10 y \\
13\end{array}$ \\
\hline & $\begin{array}{l}\text { Clasificación. Diferenciar entre los tres tipos de } \\
\text { drogas, estimulante, depresora o perturbadora, y los } \\
\text { relaciona correctamente con el tipo de droga } \\
\text { propuesto. }\end{array}$ & $\begin{array}{l}\text { Identifica } \\
\text { entre } 0 \text { y } 2\end{array}$ & $\begin{array}{l}\text { Identifica } \\
\text { entre } 3 \text { y } 5\end{array}$ & $\begin{array}{l}\text { Identifica } \\
\text { entre } 6 \mathrm{y} \\
8\end{array}$ \\
\hline & $\begin{array}{l}\text { Tolerancia, dependencia y abstinencia. Conocer el } \\
\text { significado y alcance de los términos tolerancia, } \\
\text { dependencia y abstinencia, relacionado con las } \\
\text { drogas. }\end{array}$ & $\begin{array}{l}\text { Identifica } \\
\text { entre } 0 \text { y } 1 \\
\text { término }\end{array}$ & $\begin{array}{l}\text { Identifica } \\
2 \text { términos }\end{array}$ & $\begin{array}{l}\text { Identifica } \\
\text { los } 3 \\
\text { términos }\end{array}$ \\
\hline \multirow[t]{4}{*}{$\begin{array}{l}\text { Las drogas y la } \\
\text { salud }\end{array}$} & $\begin{array}{l}\text { Prevención: conocer algunos mitos y verdades. } \\
\text { Relacionar cada uno de los } 7 \text { tipos de droga } \\
\text { propuesto, tabaco, alcohol, marihuana, cocaína, } \\
\text { éxtasis, heroína o alucinógenos, con algunos de los } \\
\text { efectos que producen sobre la salud a largo plazo. }\end{array}$ & $\begin{array}{l}\text { Identifica } \\
\text { entre } 0 \text { y } 4\end{array}$ & $\begin{array}{l}\text { Identifica } \\
\text { entre } 5 \text { y } 9\end{array}$ & $\begin{array}{l}\text { Identifica } \\
\text { entre } 10 \mathrm{y} \\
14\end{array}$ \\
\hline & $\begin{array}{l}\text { Prevención: conocer los componentes del tabaco. } \\
\text { Identificar algunos de los principales componentes } \\
\text { del tabaco. }\end{array}$ & $\begin{array}{l}\text { Marca de } \\
0 \text { a } 6\end{array}$ & $\begin{array}{l}\text { Marca de } \\
7 \text { a } 11 \text { de }\end{array}$ & $\begin{array}{l}\text { Marca de } \\
12 \text { a } 17\end{array}$ \\
\hline & $\begin{array}{l}\text { Prevención: conocer los efectos del cannabis en el } \\
\text { organismo. Identificar algunos de los principales } \\
\text { efectos del cannabis. }\end{array}$ & $\begin{array}{l}\text { Identifica } \\
\text { entre } 0 \text { y } 3\end{array}$ & $\begin{array}{l}\text { Identifica } \\
\text { entre } 4 \text { y } 6\end{array}$ & $\begin{array}{l}\text { Identifica } \\
\text { entre } 7 \mathrm{y} \\
9\end{array}$ \\
\hline & $\begin{array}{l}\text { Prevención: conocer los efectos del alcohol en el } \\
\text { organismo. Identificar las principales consecuencias } \\
\text { del alcohol en el organismo }\end{array}$ & $\begin{array}{l}\text { Detecta } \\
\text { entre } 0 \mathrm{y} \\
3 \text {. }\end{array}$ & $\begin{array}{l}\text { Detecta } \\
\text { entre } 4 \mathrm{y} \\
7 .\end{array}$ & $\begin{array}{l}\text { Detecta } \\
\text { entre } 8 \mathrm{y} \\
10 \\
\text { preguntas } \\
\text {. }\end{array}$ \\
\hline Tratamiento & $\begin{array}{l}\text { Identificar algunas de las principales pautas a tener } \\
\text { en cuenta en el tratamiento de un adicto. }\end{array}$ & $\begin{array}{l}\text { Detecta } \\
\text { entre } 0 \text { y } \\
3 \text {. }\end{array}$ & $\begin{array}{l}\text { Detecta } \\
\text { entre } 4 \mathrm{y} \\
7 .\end{array}$ & $\begin{array}{l}\text { Detecta } \\
\text { entre } 8 \text { y } \\
10 \\
\text { preguntas } \\
\text {. }\end{array}$ \\
\hline
\end{tabular}

Fuente: Elaboración propia 
ANEXO 2. Tabla de Categorías de la Narrativa.

\begin{tabular}{|c|c|}
\hline \multicolumn{2}{|r|}{ Categoría I. Papel del profesor } \\
\hline Subcategorías & Descripción \\
\hline $\begin{array}{l}\text { 1. } \\
\text { Organización } \\
\text { del tiempo }\end{array}$ & $\begin{array}{l}\text { Deseable. Gestión del tiempo en función de las necesidades del aula para sacar el máximo } \\
\text { rendimiento posible atendiendo y negociando los plazos con los estudiantes, si fuera preciso. } \\
\text { Buena. Gestión del tiempo de forma rigurosa, por lo que el tiempo ofrecido para realizar cada } \\
\text { actividad, es el misma para todos los estudiantes y los plazos de entrega solo dependerán del grado } \\
\text { de dificultad de la misma. } \\
\text { Insuficiente. Se ofrece el mismo tiempo para realizar las tareas en todo tipo de actividades y para } \\
\text { todos los estudiantes, sin conceder más plazo, aunque el alumnado intente negociar con el mismo } \\
\text { o justifique un motivo para pedir más tiempo. No escucha problemas personales }\end{array}$ \\
\hline
\end{tabular}

2

Organización

Deseable. Se produce un cambio en positivo de la metodología con respecto al trimestre anterior a de contenidos la intervención, siendo que esta nueva forma de trabajo más atractiva y menos aburrida, que lejos de ser una metodología monótona, logra motivar tanto por la metodología junto con el clima afectivo Menciona alguna actividad y algún contenido que le haya gustado.

Buena. No se producen cambios metodológicos considerables con respecto al trimestre anterior a la intervención, con lo que el alumnado no se motiva, aunque se pueda destacar alguna actividad o algún contenido más atractivo e interesante de forma puntual.

Insuficiente. La metodología usada durante la intervención, es peor que la del trimestre anterior, con resultado de un alumnado más apático, menos motivado, no encontrando ninguna actividad o contenido que les haya resultado atractivo o interesante

3. Deseable. Las clases de ciencias durante la intervención son divertidas.

Organización Buena. Las clases de ciencias durante la intervención son divertidas de forma puntual.

de actividades Insuficiente. Las clases de ciencias durante la intervención no son divertidas.

\section{Categoría II. Papel del alumnado}

Cómo trabaja Deseable. El estudiante trabaja de forma activa y dinámica en el aula durante la intervención, en el aula sintiéndose libre de dar su opinión.

Buena. El estudiante trabaja de forma activa y dinámica en el aula durante la intervención solo bajo la presión o motivación del docente, opinando solo cuando se le pregunta.

Insuficiente. El estudiante no muestra implicación en la dinámica del aula y no opina, salvo circunstancias excepcionales.

\section{Categoría III. Interrelación estudiante-docente}

Cómo interacciona

con alumnado
Deseable. El docente estimula a participar activamente a toda la clase, resolviendo los problemas surgidos con dialogo, siendo paciente cuando explica, teniendo un buen clima de aula, por regla el general.

Buena. El docente no estimula siempre a la participación activa de la clase, interviniendo en los conflictos de forma solo cuando fuera estrictamente necesario, dependiendo el clima de aula del día.

Insuficiente. El docente nunca estimula a los estudiantes a participar, no interviniendo en los conflictos salvo para sancionar alguna conducta grave, teniendo un clima de aula negativo.

\section{Categoría IV. Papel de las CCEE}

Relevancia de Deseable: El alumnado muestra interés por los contenidos trabajados en el aula a lo largo de la los contenidos intervención, siendo las clases de utilidad en su vida cotidiana. Hay varios contenidos relevantes, atractivos e interesantes para los estudiantes.

Bueno: El alumnado muestra que, a pesar de haber aprendido ciertos contenidos a lo largo de la intervención, no ha experimentado un aprendizaje útil para la vida. Hay algún contenido relevante, atractivo e interesante para los estudiantes.

Insuficiente: El alumnado no ha conseguido aprender nada y no encuentra relación con los contenidos para su vida cotidiana. No diferencia ningún contenido que le ha resultado relevante, atractivo e interesante. 